\title{
Novel ELISA for serodiagnosis of nasopharyngeal carcinoma based on a B cell epitope of Epstein-Barr virus latent membrane protein 2
}

\author{
YIQI CAI ${ }^{1 *}$, YILING SONG $^{2 *}$, DANWEI CEN ${ }^{2 *}$, CHANQIONG ZHANG $^{2}$, SHANSHAN MAO ${ }^{2}$, XIAOXIAN YE $^{2}$, \\ YIRONG XIONG ${ }^{2}$, PENGFEI JIANG ${ }^{2}$, JUN CHEN ${ }^{2}$, XIANGYANG XUE $^{2}$, LIFANG ZHANG $^{2}$ and GUANBAO ZHU \\ ${ }^{1}$ Department of General Surgery, The First Affiliated Hospital of Wenzhou Medical University; \\ ${ }^{2}$ Institute of Molecular Virology and Immunology, Department of Medical Microbiology and Immunology, \\ Wenzhou Medical University, Wenzhou, Zhejiang 325000, P.R. China
}

Received October 22, 2016; Accepted September 7, 2017

DOI: $10.3892 / \mathrm{ol} .2018 .9216$

\begin{abstract}
Epstein-Barr virus (EBV) is widespread and is associated with nasopharyngeal carcinoma (NPC). Serological detection of EBV is commonly used for screening, diagnosis and epidemiological surveys of NPC. In the present study, a novel B cell multi-epitope peptide fusion protein (EBV-LMP2-3B), which is composed of three B cell linear epitopes (RIEDPPFNSLL, TLNLT and KSLSSTEFIPN) of EBV latent membrane protein 2 (LMP2), was expressed in a prokaryotic expression system and purified using $\mathrm{Ni}^{2+}$-nitrilotriacetate-Sepharose. The immunogenicity and binding specificity of EBV-LMP2-3B were evaluated on the basis of antibody responses in immunized $\mathrm{BALB} / \mathrm{c}$ mice, western blotting and indirect immunofluorescence assay. Evaluation of EBV-LMP2-3B as a serological diagnostic reagent was performed using an indirect ELISA in 198 patients with NPC and 102 healthy adults. These results revealed that EBV-LMP2-3B was able to eliminate the high-titer serum antibody response in BALB/c mice. Western blot analysis and indirect immunofluorescence assay confirmed that the mouse immune sera recognized the native LMP2. Compared with healthy adults, patients with NPC demonstrated significantly
\end{abstract}

Correspondence to: Dr Guanbao Zhu, Department of General Surgery, The First Affiliated Hospital of Wenzhou Medical University, Wenzhou, Zhejiang 325000, P.R. China

E-mail: zgbwmc@126.com

Dr Lifang Zhang, Institute of Molecular Virology and Immunology, Department of Medical Microbiology and Immunology, Wenzhou Medical University, Wenzhou, Zhejiang 325000, P.R. China

E-mail: wenzhouzlf@126.com

*Contributed equally

Key words: Epstein-Barr virus, epitope, latent membrane protein 2, nasopharyngeal carcinoma, serological diagnosis greater reactivity to EBV-LMP2-3B $(\mathrm{P}<0.05)$. Furthermore, it was possible to effectively detect specific $\mathrm{IgG}$ in sera from patients with NPC, with a sensitivity of $91.91 \%$ and specificity of $93.14 \%$, representing an improvement over the traditional viral capsid antigen-IgA-based detection method with $59.59 \%$ sensitivity and $75.49 \%$ specificity. In conclusion, the EBV-LMP2-3B protein may be used as a serological diagnostic reagent to screen for and diagnose NPC.

\section{Introduction}

Epstein-Barr virus (EBV) is widespread among humans, with $>90 \%$ of adults latently infected with EBV throughout their lifetime (1). EBV infection may cause mononucleosis and several malignancies including nasopharyngeal carcinoma (NPC), Hodgkin's lymphoma and Burkitt's lymphoma (2-4). NPC is one of the most common malignant types of cancer in people living in the southern region of China. EBV DNA, RNA and proteins are expressed in NPC tumor cells $(5,6)$. The presence of EBV in all NPC tumor cells provides an opportunity for the development of novel diagnostic and therapeutic approaches to this type of cancer.

EBV establishes a latent infection (transformation) or undergoes lytic replication by infecting epithelial cells or B lymphocytes. Accumulating evidence suggests that carcinogenesis is principally associated with latent EBV infection $(5,6)$. During latency, all 11 late viral genes express their protein products, including 6 Epstein-Barr nuclear antigens (EBNAs 1, 2, 3A, 3B and 3C, and EBNA-LP), 2 latent membrane proteins (LMP1 and LMP2) and 2 small nuclear RNAs (EBERs) (6). Among these proteins, LMP1 is considered the primary viral oncoprotein, responsible for the malignant phenotype in NPC, whereas LMP2 maintains EBV latency (7). LMP2 localizes to cell membranes and perinuclear regions in transiently transfected cells (8). Expression of LMP2 in malignant cells and B cells suggests that LMP2 may also serve a role in cell transformation (9). Thus, LMP2 has been selected as a possible diagnostic target (10).

A number of studies have demonstrated increased levels of serum antibodies, particularly immunoglobulin (Ig)A antibody, 
against lytic and latent proteins in patients with NPC (11-13). At the time of writing, viral capsid antigen-IgA (VCA-IgA) and early antibody-IgA (EA-IgA), which are associated with NPC, are used as markers for serological diagnosis; however, neither marker is particularly specific (14). Accordingly, the aim of the present study was to develop a novel ELISA for serological diagnosis of NPC using multiple B cell epitopes of EBV LMP2.

\section{Materials and methods}

Preparation of the EBV-LMP2-3B antigen. EBV-LMP2-3B comprises three $B$ cell linear epitopes [amino acids 199-209 (RIEDPPFNSLL), 318-322 (TLNLT) and 381-391 (KSLSSTEFIPN)], which are located in the extracellular region of the LMP2 protein, with each epitope connected by a flexible peptide linker (GS), to construct a novel multi-epitope peptide, EBV-LMP2-3B. The corresponding DNA sequence was modified based on prokaryotic codon usage by Java Codon Adaptation Tool (http://www.jcat.de/), synthesized (by Shanghai Sangon Pharmaceutical Co., Ltd., Shanghai, China), and cloned into the pET32a(+) vector using BamHI and HindIII to generate pET32a(+)/EBV-LMP2-3B, which expressed a thioredoxin (Trx) fusion EBV-LMP2-3B protein with three tags (Trx, His and S) in Escherichia coli BL21(DE3) induced by $1 \mathrm{mM}$ isopropyl $\beta$-D-1-thiogalactopyranoside (Merck KGaA, Darmstadt, Germany). The successful creation of the fusion protein was verified using $12 \%$ Tricine SDS-PAGE and western blotting with horseradish peroxidase (HRP)-conjugated-anti-His monoclonal antibody (cat. no. A00174; 1:5,000; KPL, Inc., Gaithersburg, MD, USA). Following confirmation, the EBV-LMP2-3B proteins were purified using an $\mathrm{Ni}^{2+}$-nitrilotriacetate-Sepharose column (Qiagen, Inc., Valencia, CA, USA). On the basis of previous methods (15), the native LMP2 was prepared from EBV B95-8 cells (American Type Culture Collection, Manassas, VA, USA) using a membrane protein extraction kit (BestBio Biotechnology Co., Shanghai, China).

Preparation of mice immune sera. Female BALB/c mice $(\mathrm{n}=27$; mean weight, $16.12 \pm 0.25 \mathrm{~g})$ between 6 and 8 weeks of age (Shanghai Laboratory Animal Co., Ltd., Shanghai, China) were used for experiments according to approved protocols and in accordance with recommendations for the proper use and care of laboratory animals. Mice were maintained at a constant temperature $\left(22 \pm 2^{\circ} \mathrm{C}\right)$ with humidity between 40 and $70 \%$. A $12 \mathrm{~h}$ light/dark cycle was maintained and mice had free access to food and water. The mice were randomly divided into three equal groups ( 9 mice per group) and immunized with purified EBV-LMP2-3B fusion protein $(50 \mu \mathrm{g} / 100 \mu \mathrm{l})$, Trx-His-tag [pET32a(+) basal plasmid protein] $(50 \mu \mathrm{g} / 100 \mu \mathrm{l})$ or PBS $(100 \mu \mathrm{l})$ as a negative control. This was repeated three times at 2 -week intervals. Blood was collected at weeks 0, 2, 4 and 8 , and sera were removed and stored at $-80^{\circ} \mathrm{C}$.

ELISA detection. Purified EBV-LMP2-3B, native EBV-LMP2, Trx-His-tag, or synthetic peptides RIEDPPFNSLL, TLNLT and KSLSSTEFIPN $(1 \mu \mathrm{g})$ were dissolved in $100 \mu \mathrm{l}$ PBS and used to coat each well of a plate (96-well plates; Corning Incorporated, Corning, NY, USA). Plates were incubated at $4^{\circ} \mathrm{C}$ overnight. The coated plates were blocked for $1 \mathrm{~h}$ at $37^{\circ} \mathrm{C}$ with blocking buffer [5\% non-fat dry milk and $0.05 \%$ Tween-20 in PBS (PBS-T)] and incubated with serum samples (diluted 1:100) for $1 \mathrm{~h}$ at $37^{\circ} \mathrm{C}$. Following washing with PBS-T, bound antibodies were detected following incubation for $1 \mathrm{~h}$ at $37^{\circ} \mathrm{C}$ with HRP-conjugated anti-mouse $\operatorname{IgG}(1: 2,000$; cat. no. A0216; Unitech Co., Ltd., Chiba, Japan) in blocking buffer, followed by washing with PBS-T, 3,3',5,5'-tetramethylbenzidine and $\mathrm{H}_{2} \mathrm{O}_{2}$ for $10 \mathrm{~min}$ at $37^{\circ} \mathrm{C}$. Color development was determined at $490 \mathrm{~nm}$ using an ELISA plate reader (ELx800; Bio-Tek Instruments, Inc., Winooski, VT, USA). All samples were run in triplicate.

Western blot analysis. The purified EBV-LMP2-3B, Trx-His-tag and native EBV-LMP membrane protein samples were analyzed using SDS-PAGE (12\% gel) and western blotting. Rabbit serum against EBV membrane protein $(1: 5,000)(16)$, mouse immune sera against EBV-LMP2-3B fusion proteins $(1: 5,000)$ and sera from patients with NPC $(1: 5,000)$ were used as the primary antibodies. HRP-conjugated goat anti-rabbit IgG [heavy and light $(\mathrm{H}+\mathrm{L})]$ (cat. no. GAR007; ABR Inc., Fairbanks, AK, USA), HRP-conjugated goat anti-mouse IgG (H+L) (cat. no. 00001-2; ABR Inc.) or HRP-conjugated goat anti-human IgG (H+L) (cat. no. ICT-6291; 1:10,000; Thermo Fisher Scientific, Inc., Waltham, MA, USA) were used as the corresponding secondary antibodies. Antibodies were diluted in TBS with Tween-20 containing 5\% nonfat milk (w/v). The protein bands were visualized using $0.005 \%$ 4-chloro-1-naphthol and a $0.015 \%$ hydrogen peroxidase color development substrate (cat. no. 2012911; Branch of the Biological Technology Co., Ltd., Hangzhou China).

Indirect immunofluorescence assay. B95-8 cells, collected and fixed with $4 \%$ paraformaldehyde (PFA) on glass slides, were used as an antigen substrate. The slides were blocked with blocking buffer [PBS containing $5 \%$ fetal bovine serum (FBS)] at $4^{\circ} \mathrm{C}$ overnight. The cells were incubated with either immune sera against anti-EBV-LMP2-3B $(50 \mu \mathrm{g} / \mathrm{ml}$; cat. no. N29018; BestBio Biotechnology Co., Shanghai, China) or control sera (cat. no. N63728; BestBio Biotechnology Co.) diluted in PBS with 5\% FBS at a 1:100 dilution for $2 \mathrm{~h}$ at room temperature, followed by incubation with goat anti-mouse IgG fluorescein isothiocyanate (FITC)-conjugated (cat. no. D111035; Invitrogen; Thermo Fisher Scientific, Inc.) secondary antibody diluted 1:100 with PBS containing 5\% FBS for $1 \mathrm{~h}$ at room temperature. Cells were observed under a fluorescence microscope (magnification, x400).

Detection of specific Ig G antibodies in sera from patients with $N P C$. Blood samples were obtained from 198 patients with NPC and 102 healthy adults at The First Affiliated Hospital of Wenzhou Medical University (Zhejiang Province, China). Sera were separated and stored at $-80^{\circ} \mathrm{C}$ until they were retrieved for further analysis (15). All the NPC cases were confirmed by clinical and histopathological diagnoses. The present study was approved by the Human Research Ethics Committee of The First Affiliated Hospital of Wenzhou Medical University, and written informed consent was obtained from each patient. Using EBV-LMP2-3B fusion proteins, native EBV-LMP2 protein or Trx-His-tag as coated antigens, specific antibodies 
in the sera of patients with NPC and healthy individuals were detected by indirect ELISA performed as aforementioned. HRP-conjugated goat anti-human $\operatorname{IgG}(\mathrm{H}+\mathrm{L})$ diluted at 1:5,000 was used as the secondary antibody. The serum antibody titers of EBV VCA/IgA were also detected using a commercial ELISA kit (Zeus Scientific, Inc., Branchburg, NJ, USA).

Statistical analysis. One-way analysis of variance was used to evaluate the differences in the antibody levels between different groups. Multiple comparisons between the groups was performed using the Student-Neumann-Keuls method. $\mathrm{P}<0.05$ was considered to indicate a statistically significant different. All calculations were performed with the SPSS software (version 16.0; SPSS, Inc., Chicago, IL, USA).

\section{Results}

Expression and identification of EBV-LMP2-3B. The EBV-LMP2-3B fusion protein was expressed in $E$. coli BL21(DE3) of EBV-LMP2-3B and three tags, including Trx, His and $\mathrm{S}$ (Fig. 1), with an approximate molecular mass of $22 \mathrm{kDa}$, which was greater than that of the Trx-His-tag protein $(21 \mathrm{kDa})$ produced from the empty pET32a $(+)$ vector. EBV-LMP2-3B fusion protein may be specifically probed with 6x his-tag monoclonal antibody in sera from either Trx-His-tag- or EBV-LMP2-3B-immunized mice or mixed sera from 5 patients with NPC (Fig. 2A-D).

EBV-LMP2-3B-induced specific antibody responses in mice. Results revealed that EBV-LMP2-3B-specific IgG antibody levels were significantly increased compared with those in sera from either the Trx-His-tag-immunized or PBS control groups $(\mathrm{P}<0.01)$. The highest level of antibodies from EBV-LMP2-3B-immunized mice was obtained at 8 weeks post-immunization (Fig. 3A and B). The levels of antibodies against all the synthesized peptides (RIEDPPFNSLL, TLNLT and KSLSSTEFIPN) detected in the sera of EBV-LMP2-3B-immunized mice (Fig. 3C-E) were significantly increased compared with those from either Trx-His-tag-immunized mice or the PBS control group $(\mathrm{P}<0.05)$.

Immune sera against EBV-LMP2-3B recognizes native $L M P 2$ antigen. Native LMP2 antigen was detected in sera from all EBV-LMP2-3B-, Trx-His-tag- and PBS-immunized mice. The levels of specific IgG antibodies in EBV-LMP2-3B-immunized mice were significantly increased compared with those in either Trx-His-tag-immunized mice or the PBS control group at 6 and 8 weeks post-immunization $(\mathrm{P}<0.05$; Fig. $4 \mathrm{~A})$. Antibody titers were significantly increased in EBV-LMP2-3B-immunized mice compared with the control mice at week 8 (Fig. 4B). Immunofluorescence labeling confirmed that three polytopes of LMP, predominantly located on the surface of EBV B95-8 cells, were recognized by specific sera from the EBV-LMP2-3B group, as well as by the sera of B95-8 cell lysate-immunized rabbits (Fig. 4C).

Sensitivity and specificity of the EBV-LMP2-3B-based ELISA. The reactivity of sera from patients with NPC and healthy individuals was analyzed using the EBV-LMP2-3B-based

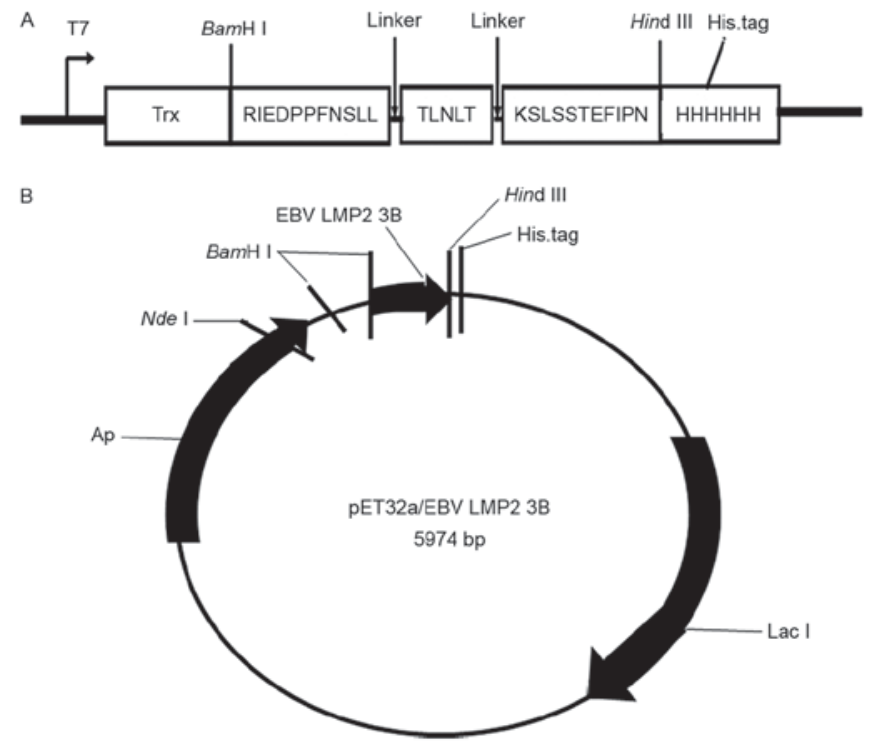

Figure 1. Construction of the pET32a(+)/EBV-LMP2-3B recombinant plasmid. The three B cell linear epitopes from the EBV-LMP2 protein (RIEDPPFNSLL, TLNLT and KSLSSTEFIPN) were linked by a flexible peptide and the whole gene sequence was optimized for prokaryotic codons, (A) synthesized, and then (B) cloned into the prokaryotic expression vector pET32a(+) between the restriction sites of BamHI and Hind $\amalg$ to construct the recombinant plasmid pET32a(+)/EBV-LMP2-3B. EBV, Epstein-Barr virus; LMP, latent membrane protein.

ELISA assay (Fig. 5). Compared with titers in healthy individuals, the titers of EBV-LMP2-3B-specific serum IgG antibodies detected in the NPC group were significantly increased $(\mathrm{P}<0.05)$. The rates of serum antibody positivity against EBV-LMP2-3B and synthesized peptides were further evaluated in 198 patients with NPC and 102 healthy subjects. Samples with optical density at $450 \mathrm{~nm}\left(\mathrm{OD}_{450}\right)$ values above the threshold (i.e. the mean \pm 2 standard deviations of the $\mathrm{OD}_{450}$ value of sera from healthy individuals) were determined to be antigen-positive. The results revealed that antibody-positivity rates against EBV-LMP2-3B and the three synthesized peptides (RIEDPPFNSLL, TLNLT and KSLSSTEFIPN) in the NPC group were 91.91 (182/198), 63.63 (126/198), 57.57 (114/198) and $61.11 \%$ (121/198), respectively. The antibody-positivity rate against EBV-LMP2-3B was significantly increased compared with those against the three synthesized peptides $(\mathrm{P}<0.05)$. When the same patients' sera were tested with an EBV VCA-IgA ELISA kit (the standard assay used for diagnosing EBV infection), the antibody-positivity rates in the NPC and healthy control groups were 59.59 (118/198) and $24.50 \%$ (25/102), respectively. This result indicates that the EBV-LMP2-3B-based ELISA was sensitive and specific for the detection of NPC (Table I).

\section{Discussion}

Serological detection of EBV has been commonly used to diagnose suspected cases of NPC and has been proposed for large-scale screenings and epidemiological surveys, as well as to monitor the recurrence and progression of EBV-associated tumors (17-19). Traditionally, serum antibody detection has been performed by either immunofluorescence labeling or immunoenzymatic assay using EBV-infected lymphocytes as 


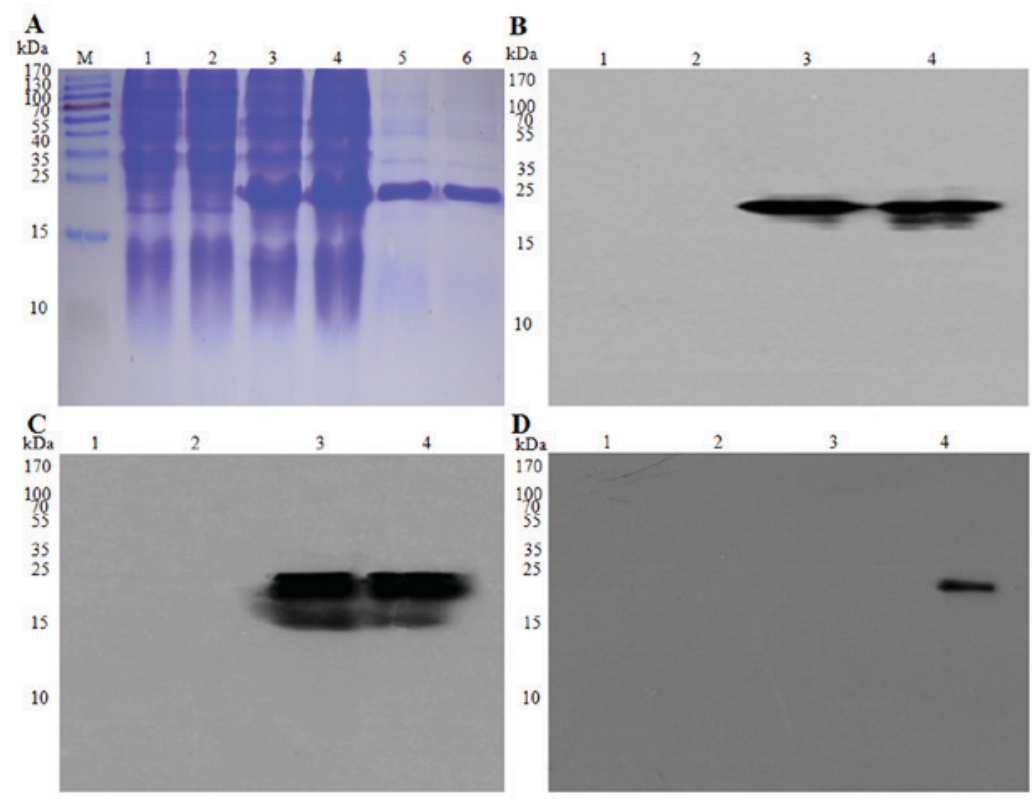

Figure 2. Expression and identification of the EBV-LMP2-3B fusion protein. EBV-LMP2-3B and Trx-His-tag protein, expressed in Escherichia coli BL21(DE3) and purified, were detected using (A) SDS-PAGE analysis and (B) western blot assay using anti-His-tag monoclonal antibody (1:5,000 dilution), (C) mouse immune serum against EBV-LMP2-3B (1:5,000 dilution), and (D) serum from patients with NPC (1:10,000 dilution). M, pre-stained protein marker, lanes 1 and 2; E. coli BL21(DE3) lysate; lane 3; pET32a(+)/EBV-LMP2-3B (Trx-LMP2-3B-His-tag; 22 kDa); lane 4, Trx-His-tag (21 kDa); lanes 5 and 6, purified EBV-LMP2-3B fusion and Trx-His-tag proteins. Epstein-Barr virus; LMP, latent membrane protein; Trx, thioredoxin.

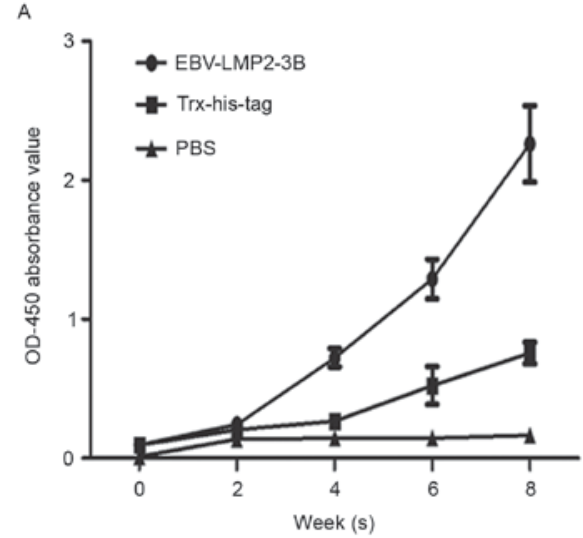

B

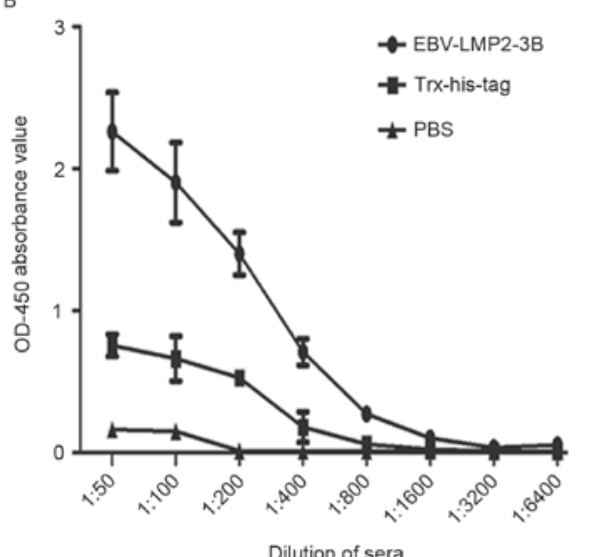

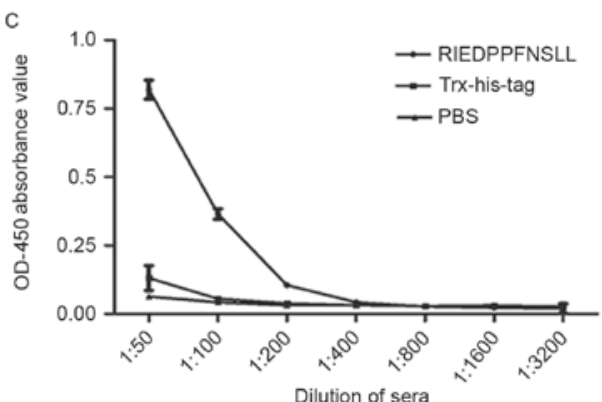

D

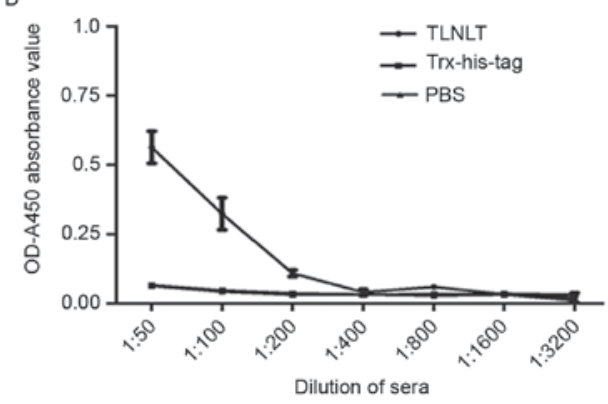

$\mathrm{E}$

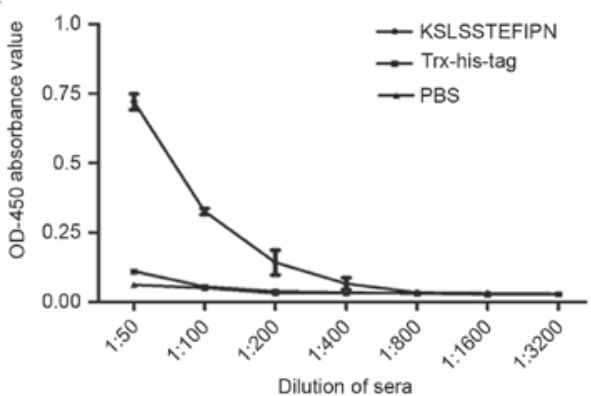

Figure 3. EBV-LMP2-3B-specific IgG in immune sera determined using an ELISA. (A) EBV-LMP2-3B-specific IgG antibodies in immunized mice, and (B) titers of specific IgG at week 8. The titers of IgG antibodies against the synthetic peptides (C) RIEDPPFNSLL, (D) TLNLT and (E) KSLSSTEFIPN at week 8. EBV, Epstein-Barr virus; LMP, latent membrane protein; Ig, immunoglobulin; OD, optical density; Trx, thioredoxin. 
A
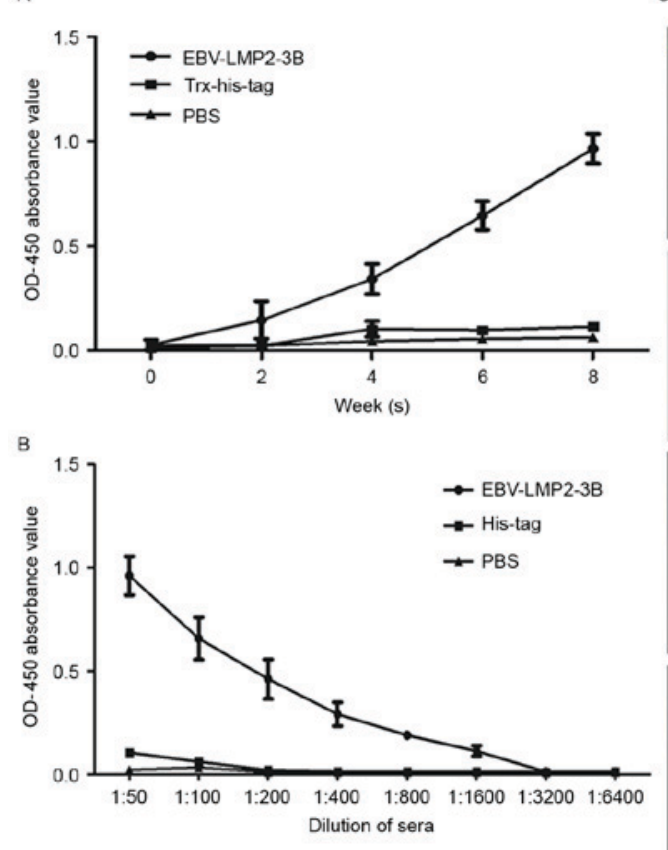

C FITC
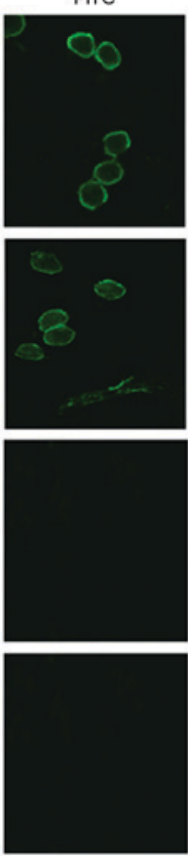
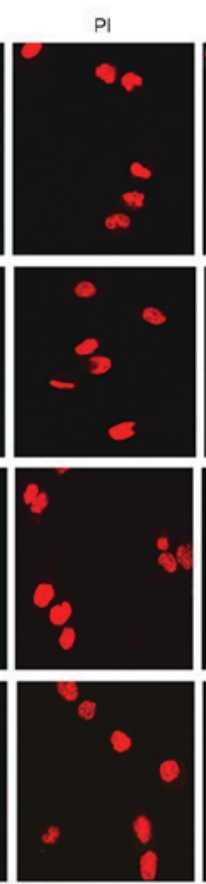

Merge
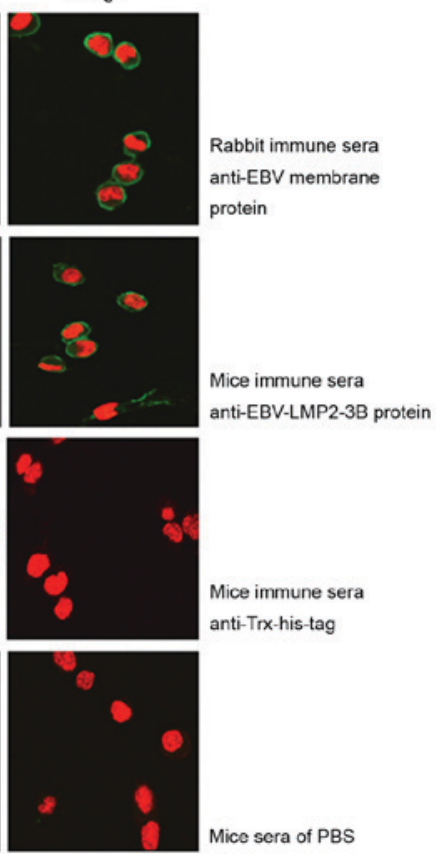

anti-EBV-LMP2-3B protein

Mice immune sera

anti-Trx-his-tag

Mice sera of PBS

Figure 4. Recognition of native LMP-2A antigen by immune sera against EBV-LMP2-3B proteins. (A) Native LMP2 protein-specific IgG levels were increased compared with those in the control group at weeks 8 and 6 (t1, 44.199; t2, 252.112; P<0.05). (B) Titers of native LMP2-specific IgG at week 8 . (C) Immunofluorescence assay analysis to determine recognition of native LMP2A antigen in B95-8 cells (magnification, x400) by immune sera against EBV-LMP2-3B. EBV, Epstein-Barr virus; LMP, latent membrane protein; Ig, immunoglobulin; OD, optical density; Trx, thioredoxin.
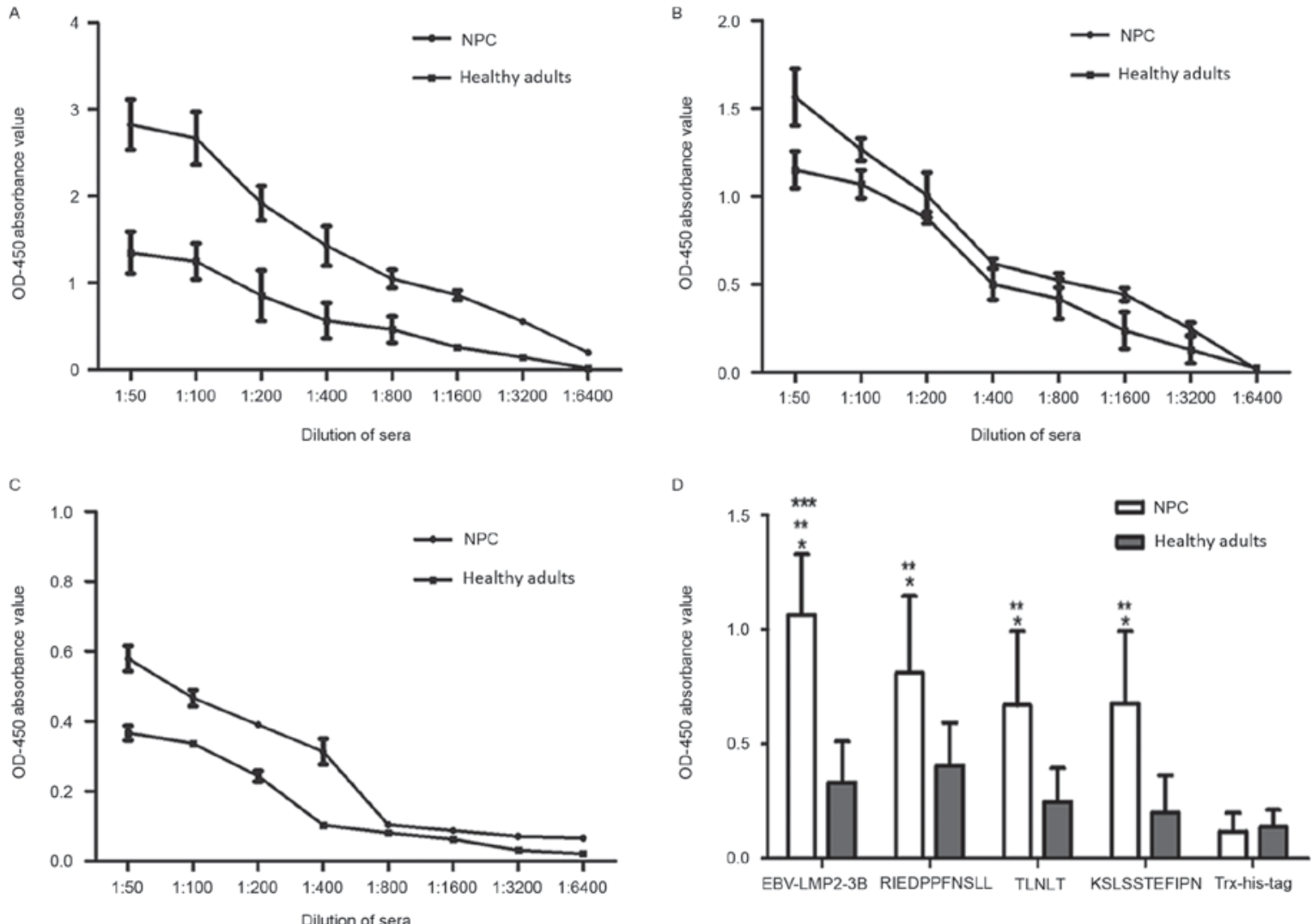

Figure 5. Reactivity of sera from patients with NPC and healthy individuals to the EBV-LMP2-3B fusion protein. The reactivity of serially diluted sera from patients with NPC and healthy individuals was determined using an indirect ELISA. Each group included 10 samples. Analysis of specificity to the (A) EBV-LMP2-3B fusion protein, (B) native EBV-LMP protein and (C) Trx-His-tag by indirect ELISA. (D) Indirect ELISA was used to evaluate the reactivity of sera diluted 1:800 from 198 patients with NPC and 102 healthy adults to the EBV-LMP2-3B fusion protein, synthesized peptides (RIEDPPFNSLL, TLNLT and KSLSSTEFIPN) and Trx-His-tag protein. Results are presented as the mean \pm standard deviation. ${ }^{*} \mathrm{P}<0.05$ compared with values from healthy controls; ${ }^{* *} \mathrm{P}<0.05$ compared with values from the group immunized with Trx-His-tag protein; ${ }^{* * * *} \mathrm{P}<0.05$ compared with the group immunized with the synthesized peptides (RIEDPPFNSLL, TLNLT and KSLSSTEFIPN). EBV, Epstein-Barr virus; LMP, latent membrane protein; Trx, thioredoxin; OD, optical density. 
the binding substrate (20). Traditional serological studies are difficult to standardize since they are semi-quantitative $(12,21)$ and are not suitable for large-scale testing or automated handling (22). At present, ELISAs are used to detect EBV-specific serum antibodies in the majority of laboratories; however, this method may overcome the shortcomings of the traditional method, and the data generated are more accurate and objective than those of earlier assays. Although VCA-IgA and EA-IgA have been widely used as serological markers of NPC in clinical screening, early diagnosis and determining the prognosis of NPC $(18,23,24)$, the VCA-IgA ELISA method has a high false positive rate (8).

A variety of EBV antigens, including those from native or recombinant EBV proteins VCA, EA, Bam HI Z EBV replication activator, EBNA1 and thymidine kinase or synthetic peptides have been used in ELISAs to screen for and diagnose NPC (25-28). We previously focused on a recombinant protein with the B cell linear epitopes of LMP2 to detect the specific antibodies in sera from patients with NPC to develop ELISAs with higher sensitivity (15), and B cell epitopes of LMP2 are optimal for use as a serological marker to diagnose NPC. In the present study, EBV-LMP2-3B was designed using three $\mathrm{B}$ cell linear epitopes (RIEDPPFNSLL, TLNLT and KSLSSTEFIPN). This EBV-LMP2-3B fusion protein expressed in a prokaryotic expression system is easily prepared and purified and demonstrates high immunogenicity. The EBV-LMP2-3B-specific antibodies in the sera of immunized mice recognize not only the native LMP2, but also the three synthesized peptides. Furthermore, immunofluorescence labeling confirmed that the antigen recognition site was located in the membrane region of B95-8 cells. The EBV-LMP2-3B protein was also recognized by the sera of patients with NPC. Taken together, these results indicate that the EBV-LMP2-3B fusion protein is highly immunogenic and that sera from immunized mice may specifically recognize and bind to native LMP2 protein. The results suggest that the EBV-LMP2-3B fusion protein may be useful as a target antigen in ELISAs to screen for antibodies against EBV, particularly in patients with NPC.

EBV-LMP2-3B-specific serum antibodies may be detected in patients with NPC, and the antibody level and positivity rate in the NPC group were significantly increased compared with those in the healthy group $(\mathrm{P}<0.05)$. Although serum antibodies specific for the synthesized peptides were also detected in patients with NPC, the antibody levels and positivity rates against EBV-LMP2-3B protein were significantly increased compared with those against each of the three synthetic peptides. Furthermore, IgG antibodies specific for EBV-LMP2-3B protein in serum specimens from 198 patients with NPC were effectively detected, representing an improvement over the traditional VCA-IgA-based assay. In addition, the antibody level and positive rate were increased compared with those against VCA-IgA. These data provide evidence that EBV-LMP2-3B is immunodominant and may be used as a target antigen in ELISAs to diagnose NPC serologically.

\section{Acknowledgements}

The present study was supported by the National Natural Science Foundation of China (grant no. 81372447). 


\section{Competing interests}

All authors declare that there are no conflicts of interest.

\section{References}

1. Niedobitek G, Meru N and Delecluse HJ: Epstein-Barr virus infection and human malignancies. Int J Exp Path 82: 149-170, 2001.

2. Hutajulu SH, Kurnianda J, Tan IB and Middeldorp JM: Therapeutic implications of Epstein-Barr virus infection for the treatment of nasopharyngeal carcinoma. Ther Clin Risk Manag 10: 721-736, 2014.

3. Niedobitek G: Epstein-Barr virus infection in the pathogenesis of nasopharyngeal carcinoma. Mol Pathol 53: 248-254, 2000.

4. Shah KM and Young LS: Epstein-Barr virus and carcinogenesis: Beyond Burkitt's lymphoma. Clin Microbiol Infect 15: 982-988, 2009.

5. Sung NS, Edwards RH, Seillier-Moiseiwitsch F, Perkins AG, Zeng Y and Raab-Traub N: Epstein-Barr virus strain variation in nasopharyngeal carcinoma from the endemic and non-endemic regions of China. Int J Cancer 76: 207-215, 1998.

6. Middeldorp JM, Brink AA, van den Brule AJ and Meijer CJ: Pathogenic roles for Epstein-Barr virus (EBV) gene products in EBV-associated proliferative disorders. Crit Rev Oncol Hematol 45: 1-36, 2003.

7. Dawson CW, Port RJ and Young LS: The role of the EBV-encoded latent membrane proteins LMP1 and LMP2 in the pathogenesis of nasopharyngeal carcinoma (NPC). Semin Cancer Biol 22: 144-153, 2012.

8. Cai WM, Li YW, Wu B, Liu YY, Hu YH, Gu XZ, Liu HY and Wang GD: Serologic diagnosis of nasopharyngeal carcinoma. A double-blind study of four EB virus antibodies with evaluation by sequential discrimination. Int J Radiat Oncol Biol Phys 9: $1763-1768,1983$

9. Pang MF, Lin KW and Peh SC: The signaling pathways of Epstein-Barr virus-encoded latent membrane protein 2A (LMP2A) in latency and cancer. Cell Mol Biol Lett 14: 222-247, 2009.

10. Straathof KC, Leen AM, Buza EL, Taylor G, Huls MH, Heslop HE, Rooney CM and Bollard CM: Characterization of latent membrane protein 2 specificity in CTL lines from patients with EBV-positive nasopharyngeal carcinoma and lymphoma. J Immunol 175: 4137-4147, 2005.

11. Chien YC, Chen JY, Liu MY, Yang HI, Hsu MM, Chen CJ and Yang CS: Serologic markers of Epstein-Barr virus infection and nasopharyngeal carcinoma in Taiwanese men. N Engl J Med 345 1877-1882, 2001

12. Ji MF, Wang DK, Yu YL, Guo YQ, Liang JS, Cheng WM, Zong YS, Chan KH, Ng SP, Wei WI, et al: Sustained elevation of Epstein-Barr virus antibody levels preceding clinical onset of nasopharyngeal carcinoma. Br J Cancer 96: 623-630, 2007.

13. Cao SM, Liu Z, Jia WH, Huang QH, Liu Q, Guo X, Huang TB, Ye W and Hong MH: Fluctuations of epstein-barr virus serological antibodies and risk for nasopharyngeal carcinoma: A prospective screening study with a 20 -year follow-up. PLoS One 6: e19100, 2011.

14. Yu KJ, Hsu WL, Pfeiffer RM, Chiang CJ, Wang CP, Lou PJ, Cheng YJ, Gravitt P, Diehl SR, Goldstein AM, et al: Prognostic utility of anti-EBV antibody testing for defining NPC risk among individuals from high-risk NPC families. Clin Cancer Res 17: 1906-1914, 2011.

15. Xue X, Zhu S, Li W, Chen J, Ou Q, Zheng M, Gong W and Zhang L: Identification and characterization of novel B-cell epitopes within EBV latent membrane protein 2 (LMP2). Viral Immunol 24: 227-236, 2011.
16. Lin X, Chen S, Xue X, Lu L, Zhu S, Li W, Chen X, Zhong X, Jiang P, Sename TS, et al: Chimerically fused antigen rich of overlapped epitopes from latent membrane protein 2 (LMP2) of Epstein-Barr virus as a potential vaccine and diagnostic agent. Cell Mol Immunol 13: 492-501, 2016.

17. Han BL, Xu XY, Zhang CZ, Wu JJ, Han CF, Wang H, Wang X, Wang GS, Yang SJ and Xie Y: Systematic review on Epstein-Barr virus (EBV) DNA in diagnosis of nasopharyngeal carcinoma in Asian populations. Asian Pac J Cancer Prev 13: 2577-2581, 2012.

18. Li S, Deng Y, Li X, Chen QP, Liao XC and Qin X: Diagnostic value of Epstein-Barr virus capsid antigen-IgA in nasopharyngeal carcinoma: A meta-analysis. Chin Med J (Engl) 123: 1201-1205, 2010

19. Ng WT, Yau TK, Yung RW, Sze WM, Tsang AH, Law AL and Lee AW: Screening for family members of patients with nasopharyngeal carcinoma. Int J Cancer 113: 998-1001, 2005.

20. Rea TD, Ashley RL, Russo JE and Buchwald DS: A systematic study of Epstein-Barr virus serologic assays following acute infection. Am J Clin Pathol 117: 156-161, 2002

21. Yi Z, Yuxi L, Chunren L, Sanwen C, Jihneng W, Jisong Z and Huijong Z: Application of an immunoenzymatic method and an immunoautoradiographic method for a mass survey of nasopharyngeal carcinoma. Intervirology 13: 162-168, 1980.

22. Stevens SJ, Zwaan CM, Verkuijlen SA and Middeldorp JM: Epstein-Barr virus (EBV) serology for predicting distant metastases in a white juvenile patient with nasopharyngeal carcinoma and no clinical response to EBV lytic induction therapy. Head Neck 28: 1040-1045, 2006.

23. Cai YL, Zheng YM, Wang W, Wei Y, Shen XX, Cheng JR, Wu YS, Gao JQ, Zhong WM and Li J: Combined detection of Epstein-Barr virus antibodies for serodiagnosis of nasopharyngeal carcinoma. Nan Fang Yi Ke Da Xue Xue Bao 30: 2746-2748, 2010 (In Chinese).

24. Sun P, Chen C, Cheng YK, Zeng ZJ, Chen XL, Liu LZ and Gu MF: Serologic biomarkers of Epstein-Barr virus correlate with TNM classification according to the seventh edition of the UICC/AJCC staging system for nasopharyngeal carcinoma. Eur Arch Otorhinolaryngol 271: 2545-2554, 2014.

25. Connolly Y, Littler E, Sun N, Chen X, Huang PC, Stacey SN and Arrand JR: Antibodies to Epstein-Barr virus thymidine kinase: A characteristic marker for the serological detection of nasopharyngeal carcinoma. Int J Cancer 91: 692-697, 2001.

26. Dardari R, Hinderer W, Lang D, Benider A, El Gueddari B, Joab I, Benslimani A and Khyatti M: Antibody responses to recombinant Epstein-Barr virus antigens in nasopharyngeal carcinoma patients: Complementary test of ZEBRA protein and early antigens p54 and p138. J Clin Microbiol 39: 3164-3170, 2001.

27. Karray H, Ayadi W, Fki L, Hammami A, Daoud J, Drira MM, Frikha M, Jlidi R and Middeldorp JM: Comparison of three different serological techniques for primary diagnosis and monitoring of nasopharyngeal carcinoma in two age groups from Tunisia. J Med Virol 75: 593-602, 2005.

28. van Grunsven WM, Spaan WJ and Middeldorp JM: Localization and diagnostic application of immunodominant domains of the BFRF3-encoded Epstein-Barr virus capsid protein. J Infect Dis 170: 13-19, 1994.

This work is licensed under a Creative Commons Attribution-NonCommercial-NoDerivatives 4.0 International (CC BY-NC-ND 4.0) License. 